International Journal of Linguistics, Literature and Translation

ISSN: 2617-0299 (Online); ISSN: 2708-0099 (Print)

DOI: 10.32996/ijltt

Journal Homepage: www.al-kindipublisher.com/index.php/ijltt

\title{
Reception of Jin Yong's Wuxia Novels in English and French: A Sentiment Analysis with Reflection
}

\author{
Kan Wu $\mathbf{8} \square$ \\ PhD and Lecturer, School of Foreign Languages, Zhejiang University of Finance and Economics Dongfang College, Zhejiang, \\ China \\ $\triangle$ Corresponding Author: Kan Wu, E-mail: wukanq@163.com
}

\begin{tabular}{|c|c|}
\hline ARTICLE INFORMATION & ABSTRACT \\
\hline Received: February 04, 2021 & \multirow{7}{*}{$\begin{array}{l}\text { This research investigates the reception of English and French translations of Jin } \\
\text { Yong's Wuxia novels through sentiment analysis --- a text mining technique which } \\
\text { helps uncover readers' opinions of these translated literary works from their online } \\
\text { reviews. The findings show that almost all of the published English/French versions of } \\
\text { Jin Yong's Wuxia novels are well received by readers in both languages in terms of } \\
\text { fictional details like "character", "plot" and "narratives", despite there are some minor } \\
\text { complains. These findings lead us to reflect on the current literary position of Wuxia } \\
\text { translations in the English and French-speaking countries, where translated Wuxia } \\
\text { works positioning as "Chinese literary classics" may partly help facilitate further } \\
\text { reception of this type of traditional Chinese literature in the West. }\end{array}$} \\
\hline ccepted: March 01, 2021 & \\
\hline Volume: 4 & \\
\hline ssue: 3 & \\
\hline Ool: 10.32996/ijllt.2021.4.3.13 & \\
\hline KEYWORD & \\
\hline $\begin{array}{l}\text { 'uxia, martial arts; literary } \\
\text { anslation; Jin Yong; sentim }\end{array}$ & \\
\hline
\end{tabular}
analysis

\section{Introduction}

With rich cultural characteristics, unique authorial styles and narratives, Jin Yong's Wuxia novels have attracted a large number of interested readers in the Greater China. In more recent years, Wuxia, one of the representatives of Chinese culture, has gone international and spread widely to English/French-speaking countries through translation. Up to 2020, there are altogether eight Wuxia translations in English and French, where they six English translations are Fox Volant of the Snowy Mountain in 1994 by the Hong Kong scholar Olivia Mok, The Deer and Cauldron in 1996 by the British Sinologist John Minford, The Book and the Sword in 2005 by the British Sinologist Graham Earnshaw, A Hero Born in 2018 by the freelance translator Anna Holmwood, A Bond Undone in 2019 and A Snake Lies Waiting in 2020 by both Holmwood and Giga Chang, a Hong Kong translator. The two French translations of Jin Yong's Wuxia novels include La Légende du héros chasseur d "aigles translated by Jiann-yuh Wang in 2004 and Le Justicier et l"aigle mythique translated by Weidong Xie in 2013.

These English and French translations, widely acclaimed since their debut, have provided us with insights into how to disseminate Chinese literature in the Western world better. In addition, as far as the study of Chinese literature translation is concerned, researchers are mainly concerned with translated works, translating strategies, translation models, and publishers' choice (Zhang, 2019). Geng (2010) pointed out that "issues such as the dissemination and reception of translated works have been ignored by some researchers for a long time, and we need to take them seriously" (p. 5). When it comes to reception of translated Chinese literature, readers' reviews can be regarded as a proper indicator, because book reviews made by readers on some book promotion websites or professional forums can illustrate the popularity and even survival of a (translated) literary work.

Therefore, this article investigates these readers' reviews the English and French translations of Jin Yong's Wuxia novels through sentiment analysis --- a text mining technique, comparing the reception of Jin Yong's Wuxia novels in the English and French worlds and reflecting on the further translation of Chinese Wuxia based on its literary position in the English/French-speaking worlds.
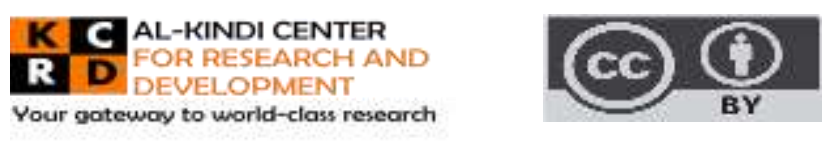

Published by Al-Kindi Center for Research and Development. Copyright (c) the author(s). This open access article is distributed under a Creative Commons Attribution (CC-BY) 4.0 license 


\section{Literature Review}

Previous studies on the reception of Jin Yong's Wuxia translations have primarily focused on cultural factors in reception, sociocultural contexts of reception, overseas disseminations of Wuxia. For the cultural concerns, Li Quan (2015), Hong (2019), Hao (2019), etc. tend to agree that translated Wuxia novels in recent years are characterized by the "Jin Yong phenomenon", which has pioneering significance for the cultural promotion of Chinese Wuxia in the West. These studies have shown the role of Jin Yong's novels in disseminating Chinese cultural in the West, inspiring Wuxia scholars at home and abroad to participate abundantly in theoretical and practical explorations for better overseas reception of this type of Chinese literature. For the sociocultural concerns, cross-cultural communication involved in the translation of Jin Yong's novels has gradually emerged. It is a new perspective to analyze the important factors in the translation of Jin Yong's Wuxia novels by referring to the mode of communication between cultures. All these concerns made scholars (e.g. Wu \& Li 2018; Hong 2019) to reflect on the present sociocultural environment for the translation and reception of Jin Yong's novels in the West in terms of ideology, poetics and patronage. For overseas disseminations, researchers (e.g. Wang 2016; Zheng 2017) in recent years began to concentrate more on overseas readers' online reviews. Their studies have demonstrated that reception research based on online reviews has two advantages: first, readers' reviews are mostly written after careful reading, so the content of the review is basically for the entire work and is relatively comprehensive; second, online readers are not easily affected by time and space when they write their reviews, and hence the review content is likely to be more thought-provoking.

While all these studies have unveiled various factors related to the ways translated Wuxia novels have been received by readers in the West, their research methodologies are mostly qualitative in nature. The present work argues that findings with stronger quantitative support are necessary for this age of digital humanities. Hence, this study will resort to sentiment analysis, a textmining technique to examine the reception of Jin Yong's Wuxia novels in English and French. Sentiment analysis, also known as supposition mining, refers to the method of extricating subjective data from a corpus through text-mining, reasoning and data classification. Through opinion examinations, it is possible to find out people's views of certain products or events, and data science researchers around the world have carried out a series of sentiment analyses based on different review data online. For instance, Aliandu (2015), Yang et al. (2020), etc. analyzed product reviews from e-commerce sites; Singh et al. (2013), Rahman \& Hossen (2019), etc. analyzed online film reviews; Neri et al. (2012), Younis (2015), etc. investigated social media data. Furthermore, Wang (2017), Chen (2020), etc. examined data from some financial documents; Huang (2019), Jing (2020), etc. analyzed course assessments. Even though areas of inquiry in these studies are diverse, they have partly illustrated the viability and potential of sentiment analyses in mining different opinions and views. Nevertheless, as most of these studies are keens on areas like finance, education, media, etc., they appear to neglect online literary reviews. Meanwhile, in a small number of studies (e.g. Zhang 2019) that do focus on literary works, the scopes and depth of their analyses are also limited. The present research argues that a sentiment analysis of online reviews could be useful for literary reception research, because these reviews are often filled with readers' stance and opinions, which are helpful to unveil the receptive status quo of a work.

As such, this study will examine the reception of Jin Yong's Wuxia fiction in Anglophone and Francophone countries through sentiment analyses. It is hoped that the results of this study can facilitate our understanding of the receptive status quo of Chinese Wuxia in the English/French speaking countries, providing some insights for the further translation of this type of Chinese literature into Western languages.

\section{Methodology}

Readers' online reviews of the six English versions and two French versions of Wuxia translations introduced in Section 1 were retrieved in the present work from the following sites: Amazon, Goodreads, Amazon.fr and Babelio, which are reputable online sites of book reviews containing large numbers of readers' reviews in English and French. Once these online reviews are obtained, the study will take the following steps in analyzing the reception of Jin Yong's Wuxia novels in English and French.

First, two corpora that contain all the English and French reviews were be separately built before the sentiment analyses. Then, the study will preprocess all the obtained online reviews, where the nltk program is utilized to perform preprocessing for cleaning the corpus, expelling stop words, and lemmatizing English and French words within the corpus. The reason for cleaning the corpus is to decrease possible negative effect of such "noise" within the corpus as emojis and additional spaces on the analyses; at the same time, excluding stop words can decrease the impact of functional words on the investigation results, and lemmatization minimize the impact (e.g. undesirable reiteration of content words) of inflectional changes in English/French on the investigation.

Second, sentiment analyses were performed on the preprocessed review corpora in English and French. In the process, the study will resort to TextBlob --- an analyzer of different sentiments in a text, loading prepared corpora of book reviews in English (3 million) and French (1 million) to train the analyzer. After numerous rounds of machine learning, the analyzer is utilized to analyze the English and French reviews to get the receptive status quo of these Wuxia translations in the two languages. Then, with the assistance of AntConc, the English and French reviews containing data with respect to characters, plots, and narratives 
will be sorted and classified. Subsequently, with both sentiment analyses and detailed semantic examinations, we can reveal the receptive status of these English and French translations of Jin Yong's Wuxia novels.

Third, the study will compare the results of reception in the two languages, in which we will manually sort the numbers of positive and negative ratings in both English and French, classifying them according to their average scores in the analysis. Then, based on the sorting and classification, we will compare possible differences and/or similarities in the receptive status quo of these translated Wuxia novels in the two languages from both holistic and local perspectives. From the holistic perspective, the average sentiment scores of each English/French translation will be compared; from the local perspective, the scores of fictional details in the same translations related to "plots", "characters" and "narratives" will be compared in a more focused way.

Finally, the study will carefully summarize all findings in the previous steps to reflect their implications for the further translation and dissemination of Jin Yong's Wuxia novels in the English/French-speaking worlds. These reflections will mostly cover the literary positions of translated Wuxia novels in the target language/cultural systems. It is hoped in this work that some of these reflections may shed new light on further promotion of Jin Yong's Wuxia novels and even the whole traditional Chinese literature in the West.

\section{Results}

For the reception based on the holistic perspective, the study finds that there are 2,644 and 1,078 positive sentiment ratings, 12 and 5 neutral sentiment ratings, and 334 and 124 negative sentiment ratings in the English and French translations of Jin Yong's Wuxia novels, respectively. When it comes to the sentiment scores, the average values of positive sentiment are 0.81 in the English translations and 0.76 in the French versions; while those of the negative sentiment are 0.13 and 0.16 separately in the English and French versions. All the details are illustrated in Table 1 and Table 2, in which we can see that Jin Yong's Wuxia stories are largely welcomed by readers of the two target languages: the content of these reviews is chiefly positive and those with a negative tone only occupy $11.1 \%$ of the total in English and $10.2 \%$ in French. Concentrating on these English and French reviews which contain 35 words or above, the study finds that they share the following points.

Table 1. Numbers the reviews in English and French

\begin{tabular}{llll}
\hline Language & Positive & Neutral & Negative \\
\hline English & 2644 & 12 & 334 \\
French & 1078 & 5 & 124 \\
\hline
\end{tabular}

Table 2. Average sentiment scores of the reviews

\begin{tabular}{lll}
\hline Language & Positive & Neutral \\
\hline English & 0.81 & 0.13 \\
French & 0.76 & 0.16 \\
\hline
\end{tabular}

First, most readers' fictional conception of these translated Wuxia works is welcomed in both English and French. These readers tend to share the view that Jin Yong's Wuxia are artworks of history, humanities and above all heroic spirits. In both English and French translations, all kinds of historic and cultural concepts make their frequent appearance, which could give readers in the two languages an experience of "brain-storming". Second, the heroic spirits denoted in the English and French versions seem to become a kind of heroic "Deja Vu" to these readers. As some readers express that while reading these Wuxia translations, they are sometimes reminded of those heroes and knights in the chivalric romance and/or modern heroic fantasies. Hence, they often compare these heroic elements in Wuxia with those in the Western heroic literature. Third, rich historical elements of ancient China included in Wuxia appear to become the shared interest for readers in both languages. Some readers noted that they rarely come across literary works outside the Western ones. Consequently, these Wuxia translations have given them a fresh experience of the Eastern heroic epics. Moreover, the readers are likely to have certain understandings of the sociocultural changes in Chinese history, which could be an extra reward to these readers as they read Jin Yong's Wuxia novels. Fourth, readers in both English and French also mentioned that they are partially dissatisfied with some of the story plots, where some pessimistic tones seem to prevail in a way of "irrelevant doomsday". This may partly discourage some readers from further reading these Wuxia translations, even though it is one of Jin Yong's Wuxia stories' distinctive components.

For the reception based on the three fictional features, the study finds that the average sentiment scores of English and French readers' reviews on the characters, plots, narratives and translating choices in Jin Yong's novels are all greater than 0 , which 
points to the positive reception. Among these fictional features, characters and narratives are the best received. Based on the results of sentiment analysis, it can be found that English and French readers generally hold a positive attitude towards the three fictional features of Jin Yong's Wuxia novels. Therefore, it is clear that English and French readers are generally satisfied with the Wuxia translations regarding the three fictional features.

According to the results of sentiment analysis, readers in both English and French show a positive attitude towards fictional features regarding characters in Jin Yong's Wuxia novels. Focusing relevant reviews in both languages, we found that most readers think that the characters in Jin Yong's novels are accessible and the emotions and inner thoughts of these Wuxia characters can be easily felt. Besides, characters in Jin Yong's novels are also rich in heroic images, with some characters carrying a strong sense of chivalry. Wuxia heroes/heroines with different worldviews in the stories make the fictional details live and vivid. Nonetheless, some readers also hold that some translated character names are hard to follow, making readers confused with the complicated interpersonal relationship between them. Moreover, some readers say that the surreal depictions of the Wuxia spirits in the novels are frustrating, since they are relatively difficult to understand from a Western perspective.

Regarding the plots in these Wuxia novels, most readers in both languages appear to positively affect the sentiment analysis results. By focusing on these plot-related reviews in both English and French, it can be found that most readers have frequently labeled the plot of Jin Yong's novel as "fascinating". Some readers say the storyline is fascinating, scintillating, and musing, while the story itself is very infectious and full of emotions. Therefore, we can see that most readers are largely satisfied with the overall plot arrangement in the English/French translations of Jin Yong's Wuxia novels. Secondly, the unique design of the story structure is also welcomed by readers in the two languages. For example, in a review for $A$ Bond Undone, some readers noted that Skyfury Guo's improvement of martial arts skills is interspersed with the progress of the main story plot, which is fresh and unique in the plot design of this kind of literature. In the reviews of The Deer and Cauldron, some readers believed that Jin Yong had combined multiple aspects of Chinese history with the story plot, which may help target readers in the two languages understand the plot against the historical backgrounds of ancient China. However, despite such a story setting being clever in the original Chinese version, some readers said that the English and French translations seem to fail in reproducing such plot design and are thus less potent to convey some of the implicit subplots the original works do. Therefore, this may give those readers in English and French a different reading experience from their counterparts in the original Chinese.

As for the story narratives, the sentiment analysis results show that most readers in two languages also take a positive attitude towards these translated Wuxia novels. Concentrating on some relevant reviews, the study finds that these readers are quite interested in Jin Yong's novels' narrative angles. The first is about the way narrative perspectives in the Wuxia novels are shifting. Many readers in English and French believe that constant shifting of narrative perspectives by Jin Yong in his Wuxia stories is innovative and successful since it provides readers with a new way to understand the Chinese thinking patterns. This kind of perspective setting is unprecedented: the continuous conversion of the narrative perspective is like a revolving camera, where panoramic views show the psychological development of those martial arts heroes and heroines in the stories. At the same time, the use of different narrative techniques and angles also makes the whole story alive. In this regard, some readers think that the varied narrative perspectives for different gender roles in these Wuxia translations may give them quite a fresh reading experience. Furthermore, many readers also mentioned that Jin Yong often narrates from a critical perspective in some novels, which may add some depth to the story itself.

\section{Discussion}

The sentiment analyses based on readers' reviews have shown that, despite there are some negative reviews, the translations of Jin Yong's Wuxia novels are, on the whole well received by readers in both English and French. However, a careful reading of all these reviews seems to show the other side of the receptive picture: the translation of Jin Yong's novels has been progressing slowly in both English and French speaking worlds; meanwhile target readers of these Wuxia translations are mostly Asian readers speaking the two languages and/or English/French people with a background in Chinese learning or Sinology. Hence, it is necessary to reflect on issues related to the "literary positioning" of and cultural elements in Jin Yong's Wuxia novels as we translate and introduce them into the English/French cultural systems.

The translation and dissemination of Chinese literature in the West generally suffers from the dilemma of marginalization of public reading. This is often attributed to differences between China and the English/French-speaking worlds in terms of history, society, and politics. But for Chinese Wuxia novels, there are still one challenge in the English and French translation of Jin Yong's novels, in which the core problem lies in the disparity between the "literary positioning" of these novels in the source (Chinese) and target languages (English/French) systems. Since the $21^{\text {st }}$ Century, Jin Yong's Wuxia novels have gradually entered a new stage of classic interpretation. The pursuit of ideology and culture in Jin Yong's works has become a widespread concern in academia. As Tang (2019: 156) said: "Jin Yong's Wuxia is a fictional incarnation of the traditional Chinese cultural system, where the interpretation of human nature is about an understanding of politics and national identities in the Chinese secular cultural systems." Domestic researchers in the field of modern and contemporary literature have continued to pay attention to the strong 
cultural interpretation in Jin Yong's Wuxia novels, combining the ideas of Confucianism, Buddhism, Taoism, and Mohism to explore the connotation of these novels. Considering this, Jin Yong's novels, which are both readable and ideological, are a feasible way to help Western readers understand the core of traditional Chinese philosophy and promote Chinese culture overseas." Furthermore, because of the cultural differences between the East and the West, the choice of cultural translation strategies in the English/French translations of Jin Yong's novels and the effectiveness of readers' reception of the cultural information have always attracted due scholarly attention. However, in the English/French-speaking worlds, Jin Yong's Wuxia novels are often labeled by the mainstream media as a "best-selling/popular novelist", meanwhile some scholars of English/French literary research even place these translated novels within a relatively single framework of "popular literature", which is clearly not on the same par with the status of "serious literature" Jin Yong's Wuxia novels have gained in the Chinesespeaking world. In addition, the need for entertainment in today's mass culture has been mainly met by the derivative products of Jin Yong's novels in the form of popular cultures - movies, games, animations, etc., which is likely to weaken the cultural details depicted in the original novels.

Furthermore, in today's globalized cultural context, it is necessary to rethink the literary positioning and target readers of Jin Yong's Wuxia novels as we promote them overseas through translation. The refinement of the translation between business/academic and public/elite determines the strategies of cultural translation and its depth of cultural interpretation, as well as the reader's reception of the translation. As the English/French translations of Jin Yong's Wuxia novels have received increasing attention, the academics have noticed the problems of its cultural positioning and target reader groups. Therefore, starting from the reality in English/French-speaking worlds, further translation of Jin Yong's Wuxia novels should consider the positioning of this type of traditional Chinese novel as "literary classics".

First of all, the positioning of the so-called "literary classics" is not based on literary criticism of elite literature/popular literature. Instead, it emphasizes the classics status of Jin Yong's novels when we translate and disseminate them in the English and French speaking worlds. This means that these translated Wuxia novels are meant to be regarded by their target readers in both languages as serious classics rather than books of entertainment. From the perspective of being a reader, French and English people generally maintain reading their existing habits (especially paper books), where they value literary works that are poetic, ideological, and pluralistic. In 2018, Anna Holmwood's English translation of "The Legend of the Condor Heroes: A Hero Born" received favorable responses from her target readers. Her translation, positioned as a fantasy literature by British publishers, is sometimes compared to the Chinese version of The Lord of the Rings and Game of Thrones. Yet, when it comes to French literary markets, such "best-selling" business model in the English-speaking world is more difficult to replicate in France, as this bestselling literature in the English-speaking world has not been popular in France. Among the existing translations of foreign literature in France, Chinese literature occupies only a marginalized niche, despite important French publishing houses such as Gallimard, Les Belles Lettres, Seuil, Philippe Biquier (Philippe Picquier), etc., have published specialized serials of Chinese literature translations, ranging from classic classics to contemporary novels. Of these specialized serials, almost all of them are related to academic research, while few are published out of concern for market popularity. Therefore, from this point of view, an emphasis on the profoundness and academic values in the translation of Jin Yong's Wuxia novels is more likely to give these translated works a proper literary position among French readers.

Furthermore, in terms of the generic characteristics of Wuxia novels, readers in both English and French face three thresholds of reception: first, they do not understand the typological characteristics of traditional Chinese Wuxia literature, and so it is difficult for them to adapt to relatively fixed plot patterns and narrative rhythms. Also, because these Wuxia novels contain a lot of traditional Chinese cultural elements in relation to social customs, daily utensils, and emotional patterns, the absence of annotations and explanations would give target readers of Wuxia translation a hard time in overcoming these cultural barriers while reading. Besides, for Western readers who have never heard of Jin Yong's Wuxia novels, they are prone to regard them as fantasy literature, and thus ignoring authors' effort on specific integrations of Chinese tradition and modernity into these Wuxia works. In addition, the moral standards (such as filial piety, loyalty, righteousness, etc.) that are valued and cherished by Wuxia heroes and heroines in the stories are mostly based on the traditional Chinese patterns of ethical relations, which have a far psychological distance from contemporary readers in English and French who emphasize individual independence. Therefore, it is necessary to include some supplementary cultural notes in the translations about the cultural backgrounds and ideological connotations of Wuxia novels.

Secondly, the current literary positioning of the French/English translations has inflicted some uncertainty in terms of cultural interpretation in translation. Judging from the current translations, several translators are able to be faithful to the original work through the use of translation skills that values a balance between faithfulness to the original and smoothness in target language expression. For smooth and accurate translation, the biggest challenge is at the level of cultural translation. There is a seemingly contradictory phenomenon: translators in China tend to be "domesticated" while retaining some important cultural concepts in the novel by sticking the translated text as close to the target language in terms of idiomatic expressions as possible. By contrast, translators in the West seem to pay more attention to "foreignization" through a deviation from the mainstream values of the 
target culture by retaining those of the source culture. This is quite consistent in some translations by those Western translators, whether it is in their shared use of the spelling system that stays closer to Chinese pronunciation or an inclusion in their translations a systematic and detailed introduction to Taoist thought and theories of traditional Chinese medicine. In addition, different translation strategies actually reflect the demands of translators with different cultural identities: outsiders are eager to be understood and accepted more smoothly, while the recipient is eager to see the original as much as possible. In fact, foreignization and domestication as translation strategies have only pointed out the two ends of cultural translation. From a specific discussion of translation in some previous research, it can be seen that the translators of these English and French versions of Jin Yong's Wuxia novels have adopted these two methods comprehensively. They try to emphasize the cultural connotations in Jin Yong's Wuxia novels to varying degrees, but their cultural interpretation is limited in breadth and depth. In terms of breadth, the translator has to weaken certain cultural symbols and reduce annotations at the expense of text length in order to make the novel as easy to read as possible. In terms of depth, although the translators have introduced information such as "Wuxia", "Jianghu" and related historical background in their English and French translations in the form of prefaces or appendices, they are only simple introductions lacking necessary accuracy. As a consequence, it is especially difficult for these translators to tell their target readers the modern value of Jin Yong's novels and the deep cultural reasons why they are popular in Asia. Taking The Legend of the Condor Heroes: A Hero Born as an example, if Guo Jing's cultural personality is not explained, ordinary readers in English and French will easily regard him as an honest but dull protagonist. As an alternative, in-depth text interpretation or novel introduction may seem to challenge readers' reading patience, but they are more likely to improve target readers' comprehension of a Wuxia translation.

Finally, the research tradition of Sinology in both English and French speaking worlds has provided a paradigm and possibility for the "top-down" translation mode of Jin Yong's novels to English and French readers. As the countries with the longest history of Oriental and Chinese studies in Europe, both Britain and France attach great importance to translation theories and practices in their academic traditions, retaining the concept of heterogeneity in understanding different types of source texts. In today's French and French translation of foreign literary works, the mainstream concept is to avoid abridged translation or drastic deletion of the original text. Different from Western Sinologists in the 19th century who have little interest in the exploration of Chinese literature, contemporary Sinologist in both English and French-speaking worlds have expanded their research interests to a wider range of literary texts; and among which, traditional literature from China and other Eastern worlds is particularly valued. It is worth noting that translation and academic research French Sinology are often done simultaneously by scholars, such as André Lévy, who translated Jin Ping Mei, Journey to the West, Strange Stories from a Lonely Studio as well as a large number of Su Shi's works. Other examples include Stéphane Feuillas, a scholar of philosophy of Song Dynasty and Isabelle Rabut, a dedicated scholar of modern and contemporary Chinese literature. As most of these sinology scholars are both translators and literary researchers, the translation (especially the translation of classic works) is often accompanied by a long introduction written by the translator or other well-known scholars before the main text. This helps readers or researchers understand such information related to the source texts as the original author, generic characteristics, era background and ideological connotation. This "guided reading mode" is especially suitable for the English and French translations of Jin Yong's Wuxia novels. Publishers may also consider adopting a mode where scholars write guided readings, while translators specialize in translating work.

\section{Conclusion}

This research investigates the reception of English and French translations of Jin Yong's Wuxia novels through sentiment analysis. Through sentiment analyses, this study shows that readers of Jin Yong's Wuxia novels in English and French are largely satisfied with the translations in terms of such fictional details like characters, plots and narratives, despite there are also some minor dissatisfactions with some of these fictional features. These findings may reflect the literary positioning of the Wuxia translations in the English and French speaking worlds. Regarding this, the present study maintains that we strengthen the depth of literary/cultural interpretation in the English/French translation of Jin Yong's Wuxia novels, so as to gradually arouse readers' interest in Wuxia novels, helping them appreciate philosophies and values of traditional Chinese literature. As a representative of Chinese heroic literature, the literary vitality of Jin Yong's Wuxia novels is deeply rooted in the cultural taste of refined heroic spirit from the East. Hence, translations of Jin Yong's Wuxia novels into English and French can offer readers in the West fresh perspectives of the traditional Chinese heroic literature.

On the other hand, it is worth mentioning that there are some limitations of the present work and one of which appears to be the limited number of Wuxia translations included in the analyses and discussion. Yet, this problem can be solved in the further study when more English and French translations of Jin Yong's Wuxia novels are published and become publicly available in the reading market. Finally, this research also shows that sentiment analysis provides a new perspective and method for the research on reception of translated Chinese literature, providing a reliable tool for the evaluation of relevant empirical data related to Chinese literary translations. 


\section{Sponsoring information}

A Project Supported by Scientific Research Fund of Zhejiang Provincial Education Department (Y202044693)

\section{References}

[1] Aliandu, P. (2015). Sentiment analysis to determine accommodation, shopping and culinary location on foursquare in Kupang City. Procedia Computer Science, 72, 300-305.

[2] Chang, G. (2019). A Bond Undone: Legends of the Condor Heroes II. New York: ST. Martin's Griffin.

[3] Chen, T. (2020). Research on financial text sentiment analysis based on attention mechanism. Information Technology and Informatization, (1): 175-177.

[4] Earnshaw, G. \& Cha, L. (2004). The Book and the Sword: A Martial Arts Novel. Oxford: Oxford University Press.

[5] Geng, Q. (2010). Literary Translation and Chinese Literature "Going to the World". Shanghai: Shanghai International Studies University.

[6] Minford, J., \& Cha, L. (1997). The Deer and the Cauldron. Oxford: Oxford University Press.

[7] Mok, O. \& Yong, J. (1993). Fox Volant of the Snowy Mountain. Hong Kong: The Chinese University Press.

[8] Neri, F., Aliprandi, C., Capeci, F., Cuadros, M., \& By, T. (2012, August). Sentiment analysis on social media. In 2012 IEEE/ACM International Conference on Advances in Social Networks Analysis and Mining (pp. 919-926). IEEE.

[9] Hao, L. (2019). Film Adaptation of Louis Cha's Martial Arts Novels and Their Overseas Translations. Film Review, (16), 17.

[10] Holmwood, A. (2018). A Hero Born: Legends of the Condor Heroes. London: MacLehose Press.

[11] Holmwood, A \& Chang, G. (2020). A Snake Lies Waiting: Legends of the Condor Heroes III. New York: ST. Martin's Griffin.

[12] Hong, J. (2019). An Empirical Study on the English Translation of Verbs in Jin Yong's Novels: Based on a Corpus Investigation. Fujian Forum (Humanities and Social Sciences Edition), (7), 12.

[13] Huang, Y. (2019). Research on sentiment analysis of teaching reviews based on association mining. [Master dissertation] Jiangxi University of Finance and Economics.

[14] Li, Q. (2015). Translation and Research of Jin Yong's Martial Arts Novels in the English World. Guizhou Social Sciences, (6), 96-101.

[15] Rahman, A., \& Hossen, M. S. (2019, September). Sentiment analysis on movie review data using machine learning approach. In 2019 International Conference on Bangla Speech and Language Processing (ICBSLP) (pp. 1-4). IEEE.

[16] Singh, V. K., Piryani, R., Uddin, A., \& Waila, P. (2013, March). Sentiment analysis of movie reviews: A new feature-based heuristic for aspectlevel sentiment classification. In 2013 International Mutli-Conference on Automation, Computing, Communication, Control and Compressed Sensing (iMac4s) (pp. 712-717). IEEE.

[17] Tang, Z. (2019). The Cultural Interpretation of Jin Yong's Novels and the Construction of Cultural Personality. Shanghai Culture, 8: 31-39.

[18] Wang, B. (2016). Evaluation and reception of Yan Lianke's novel "Shou Huo" in the English world: based on an investigation of English book reviews. Southern Literary Circle, 5.

[19] Wang, J. (2004). La Légende du héros chasseur d 'aigles. Paris: Librairie \& Edition You-feng.

[20] Wang, P. (2017). Application of Sentiment Analysis in Financial and Marketing Information Retrieval. Business Economics, (8), $166-167$.

[21] Wu, K, \& Li, D. (2018). Lexical Normalization in English Translations of Jin Yong's Martial Arts Fiction: A Corpus-based Study. Asia Pacific Interdisciplinary Translation Studies, (7), 93-106.

[22] Xie, W. (2013). Le Justicier et l'aigle mythique. Paris: Librairie \& Edition You-feng.

[23] Yang, L., Li, Y., Wang, J., \& Sherratt, R. S. (2020). Sentiment analysis for E-commerce product reviews in Chinese based on sentiment lexicon and deep learning. IEEE Access, 8, 23522-23530.

[24] Younis, E. M. (2015). Sentiment analysis and text mining for social media microblogs using open-source tools: an empirical study. International Journal of Computer Applications, 112(5).

[25] Zhang, L. (2019). Looking at overseas readers' reception and evaluation of Chinese translated literature from the perspective of python sentiment analysis: Taking the English translation of "Santi" as an example. Foreign Language Studies, (4): 80-86.

[26] Zheng, Y. (2017). Viewing the effect of Chinese literature translation and introduction from readers' responses: Taking the English version of "Chinese Literature" as an example. Chinese Comparative Literature, 1. 